
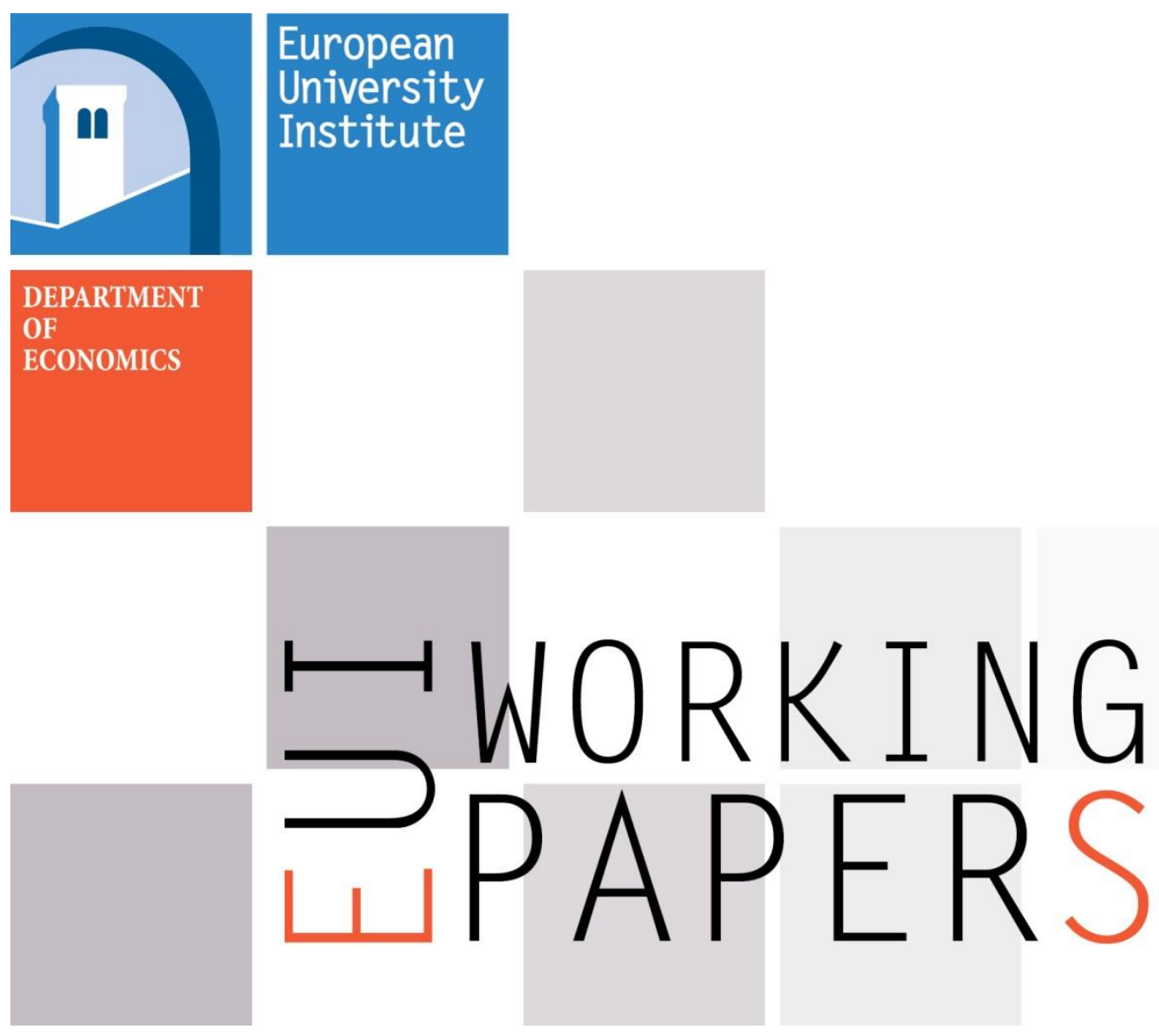

ECO 2015/01

Department of Economics

\title{
FINANCIAL CONTAGION IN NETWORKS
}

Antonio Cabrales, Douglas Gale and Piero Gottardi 

European University Institute

Department of Economics

\section{Financial Contagion in Networks}

Antonio Cabrales, Douglas Gale and Piero Gottardi

EUI Working Paper ECO 2015/01 
This text may be downloaded for personal research purposes only. Any additional reproduction for other purposes, whether in hard copy or electronically, requires the consent of the author(s), editor(s). If cited or quoted, reference should be made to the full name of the author(s), editor(s), the title, the working paper or other series, the year, and the publisher.

ISSN 1725-6704

(C) Antonio Cabrales, Douglas Gale and Piero Gottardi 2015

Printed in Italy

European University Institute

Badia Fiesolana

I - 50014 San Domenico di Fiesole (FI)

Italy

www.eui.eu

cadmus.eui.eu 


\title{
Financial Contagion in Networks*
}

\author{
Antonio Cabrales \\ University College London \\ Douglas Gale \\ Imperial College London \\ Piero Gottardi \\ European University Institute
}

This version: February 28, 2015

\begin{abstract}
This paper provides an introduction to the literature on financial contagion in networks. In the first part, we consider contagion via transmission of shocks, i.e. an abrupt drop in the flow of revenue to one firm, which affects other firms connected to it through financial linkages. We then study informational contagion, by which we mean the process whereby a shock to one market is transmitted to other markets by means of information revealed in the first market.
\end{abstract}

\section{Introduction}

The aim of this chapter is to provide an introduction to the literature on financial contagion in networks. We aim to do this by focusing on a limited number of papers in some formal detail, trying to illustrate their analogies and differences as much as possible within a common framework. We divide the discussion in two parts. In the first one, we consider contagion via transmission of shocks, i.e. an abrupt drop in the flow of revenue to one firm, which affects other firms connected to it through financial linkages. We then study informational contagion, by which we mean the process whereby a shock to one market is transmitted to other markets by means of information revealed in the first market.

In the first part we will consider mostly symmetric firms, connected via financial linkages, and symmetric networks of different kinds. The symmetry makes the analysis simpler and easier to present and it also shows that systemic problems can arise even with symmetry. This is important to note because much of the popular discussion about financial firms has been directed at firms with "special" features, such as "too-big" or "too-interconnected" to

*Prepared for The Oxford Handbook on the Economics of Networks, Yann Bramoullé, Andrea Galeotti, and Brian Rogers (Eds), Oxford University Press. We wish to thank the Editors as well as Daron Acemoglu, Alireza Tahbaz-Salehi, Matt Jackson, Matt Elliott and Ben Golub for very helpful comments and discussions on an earlier version of this paper. 
fail. We also mention the extension of the results to more general kinds of networks, and to heterogeneous firms. And we end by describing in less detail the results in a wider class of papers.

The second part analyzes informational contagion. It is divided in two subsections: the first one studies contagion between markets, the second one contagion between financial firms.

Informational contagion is in some cases related to contagion occurring through tranmission of shocks but it often amplifies the effect of the former. For example, imagine that a shock can travel in a network through a path of at most length $k$. Then, if the financial network and the origin of a shock are common knowledge among all market participants, and if $k$ is small with respect to the average network distance, the effects of a shock will be in general quite limited. But with imperfect information about the origin of a shock and the network topology, a majority of firms could optimally take protective measures to avoid contagion once a shock arrives, something they would not do with complete information.

Informational contagion can also be independent from shock transmission. This happens, for example, when a price movement in one country affects prices in a different country for informational reasons. This is because traders in the latter country can infer some information related to common shocks from the price movements in the former.

\section{Contagion through shock transmission}

\subsection{The model}

Let there be $N$ financial firms (say, banks). Each firm has liabilities, equal to $l$ towards external investors, and assets, given by claims to the returns on "projects". There are $N$ projects and the return on each project $i$ is subject to shocks: it is equal to $R$ if there is no shock, and to $R-s_{i} \in[0, R)$ if a shock hits. The analysis investigates the effects of the presence of financial linkages among firms on their financial situation, and in particular on their solvency. We can portray these linkages in a general, abstract way by saying that the value $v_{i}$ of the assets of a firm $i$ may be related to the value $v_{j}$ of the assets of any other firm $j \neq i$ as they both depend on the vector $\mathbf{r}$ which describes the realizations of the returns of the $N$ projects (for each $i, r_{i}=R-s_{i}$ if a shock hits the return of project $i$ and to $R$ otherwise). This relationship is modeled as follows:

$$
v=f(A ; \mathbf{r})
$$

where $f: \mathbb{R}_{+}^{N \times N} \times \mathbb{R}_{+}^{N} \rightarrow \mathbb{R}_{+}^{N}$ and $A$ is a $N \times N$, non negative matrix with generic entry $a_{i j}$. The matrix $A$ describes the pattern of the linkages among the $N$ firms, and the function $f($.$) the effect of these linkages on the value of the firms' assets.$

If the value $v_{i}$ of the assets of firm $i$ is lower than the value $l$ of its liabilities, the firm defaults. Because of the presence of linkages among firms, default events are correlated. 
Since default of a firm is costly (because of the destruction in value of the firms' assets due to the termination of its activities or to liquidation $\operatorname{costs}^{1}$ ), one of the main objectives of the analysis is indeed to analyze the extent of default in the system and whether generalized default, or contagion, may occur in the presence of such linkages.

In the literature we find different microfoundations for the map $f($.$) and the matrix A$, leading to different interpretations of the elements of this matrix and to different properties of $f($.$) . In particular, we present in what follows some alternative microfoundations that$ lead $f($.$) to be a linear function of \mathbf{r}$, or nonlinear but still continuous, or even discontinuous, and we will examine the consequences of these different properties.

In these microfoundations, the linkages among firms may have a different nature. In particular, in Cabrales, Gottardi and Vega Redondo (2014) [CGV] and Elliott, Golub and Jackson (2014) [EGJ] they arise from the mutual ownership of the claims to the returns of the underlying projects: that is, the returns on the assets of a generic firm $i$ are given by a certain linear combination of the returns of the $N$ projects, with weights given by the $i$-th row of the matrix $A$ : $\sum_{j} a_{i j} r_{j}$. In CGV the terms $a_{i j}$ describe the ownership by firm $i$ of claims entitling the owner to a fraction of the returns of project $j$. These claims are obtained via a sequence of rounds of exchanges of assets by each firm $i$, initially endowed with full ownership of project $i$, with a subset of other firms (constituting its immediate neighbors). The pattern of exchanges at each round is described by the matrix $B$, where the nonzero elements of row $i$ describe $i$ 's trades with its immediate neighbors. Hence we have (when the number of rounds of these exchanges is given by $K$ )

$$
A=B^{K} \text { and } f(A ; \mathbf{r})=A \mathbf{r} .
$$

EGJ, on the other hand, consider a situation where firms engage in exchanges of equity among them (again starting from a situation where each firm $i$ fully owns project $i$ ). Letting $c_{j i}$ denote the fraction of the outstanding equity of firm $i$ sold to firm $j$, and $\hat{c}_{i i}$ the fraction that remains owned by external investors, we have $\hat{c}_{i i}=1-\sum_{j \neq i} c_{j i}$. As shown by EGJ (in line with Brioschi, Buzzacchi, and Colombo (1989) and Fedenia, Hodder, and Triantis (1994)), this ownership structure again entitles the owners of equity of the $N$ firms to a linear combination of the returns of the underlying projects with weights given by the matrix

$$
A=\widehat{C}(I-C)^{-1}
$$

where $\hat{C}$ is the diagonal matrix with generic entry $\hat{c}_{i i}$ and $C$ the matrix with generic entry $c_{i j}$ (and zero diagonal terms). The fact that the mutual ownership takes now the form of equity and that, as we said above, the default of a firm entails a cost, implies that any other firm that is owning equity of the bankrupt firm must bear part of this cost, in proportion to

\footnotetext{
${ }^{1}$ We can think of $r$ and $l$ as revenues and payments due the subsequent period. The firm's assets generate revenue also in later periods, but when liquidated the value of the firm's assets is zero, for simplicity (in any case lower than the present value of future returns, hence the cost of default).
} 
the level of its equity ownership. Hence, letting $\beta$ denote the (immediate) cost of default ${ }^{2}$ for a firm, we have:

$$
v=f(A ; \mathbf{r})=A\left(\mathbf{r}-\beta \mathbf{1}_{\left\{v_{i}<l\right\}}\right),
$$

where $\mathbf{1}_{\left\{v_{i}<l\right\}}$ denotes (with a slight abuse of notation) the vector of indicator functions taking value 1 if $v_{i}<l$ and 0 otherwise, for $i=1, . ., N$. We see from (3) that, as long as $\beta>0$, the value $v$ of the firms' assets is now a discontinuous function of $\mathbf{r}$, that jumps whenever some firm defaults. Moreover, $v$ is determined as a fixed point of the map defined by (3), since the level of $v$ also determines whether a firm is solvent or not; if insolvent a firm has to pay the additional default cost, which in turn affects the value of $v$, and so on. To see this, take $N=2$,

$$
A=\left[\begin{array}{ll}
0.7 & 0.3 \\
0.3 & 0.7
\end{array}\right],
$$

$R=1$ if no shock hits and $r_{i}=0.5$ if a shock hits, $l=0.8$ and $\beta=0.5$. Then, if project 2 is hit by a shock $\left(r_{2}=0.5\right)$, not only firm 2 defaults, but also firm 1 , because of the fraction of default costs it must bear. In contrast, if $r_{i}=R$ for each $i$, there are two fixed point solutions of (3), one where both firms are solvent, and the other where both firms default. That is, simply the belief that a firm may default suffices to generate losses in the firms' value that trigger a default even when the returns on the underlying projects have not been hit by any shock.

As we have seen, the approach in CGV and EGJ looks at the presence of linkages among the assets of the different firms given by cross holdings of claims to the returns on projects. $^{3}$ In contrast, Glasserman and Young (2015) [GY] and Acemoglu, Ozdaglar and Tahbaz-Salehi (2014) [AOT] consider linkages among both assets and liabilities of firms, arising from mutual lending and borrowing relationships among them, via standard debt contracts. In this case $a_{i j}$ denotes the payments due from firm $i$ to firm $j$. The value of firm $i$ 's liabilities is then augmented now to include the value of the payments due to other firms, $l_{i}+\sum_{j \neq i} a_{i j}$. Firm $i$ is again endowed with a $100 \%$ share of the returns generated by project $i$. The book-value value of its assets is similarly augmented by the book-value of the loans it granted, $r_{i}+\sum_{j \neq i} a_{j i}$. To establish the solvency of firm $i$ however what matters is not the book-value but the actual value of the firm's assets, which reflects the actual payments made by its debtors and may be less than the due payments when they are in default. Hence the actual payments to firm $i$ depend on the value of the assets of this firm's debtors, that is on the payments they in turn receive from their own debtors. This creates an interdependence among the actual value of the assets of all firms in the system. ${ }^{4}$

\footnotetext{
${ }^{2}$ Strictly speaking, in line with footnote $1, \beta$ should be viewed as the part of the cost that must be born immediately, i.e. in the subsequent period. Also, a limited liability constraint could be added in (3) with no change in the substance of the results.

${ }^{3}$ These claims could also be given by derivatives whose return is a more general, non linear function of the yield of these projects.

${ }^{4}$ Such an environment is also considered by Shin (2009) to study the effects of changes in credit volumes.
} 
AOT and GY resort to the analysis of Eisenberg and Noé (2011) to find a consistent set of payments which can be made by firms linked by mutual borrowing and lending relationships when some of them default, while respecting the possible differences in the seniority of the firms' liabilities. Suppose that all the liabilities among firms have equal seniority $^{5}$ so that the total payments made by any given firm to other firms are distributed to them pro rata, on the basis of the value of their claims towards this firm. For any vector $\mathbf{r}$ of realized returns, a final (equilibrium) repayment vector is then obtained as a solution of the following system:

$$
p_{i}(\mathbf{r})\left(\sum_{j \neq i} a_{i j}+l\right)=\left(\sum_{j \neq i} a_{i j}+l\right) \wedge\left(\sum_{j \neq i} p_{j}(\mathbf{r}) a_{j i}+r_{i}\right)
$$

for all $i$. The actual payments made by firm $j$ to firm $i$ are then

$$
p_{i j}(\mathbf{r})=p_{i}(\mathbf{r}) a_{i j}, \text { for all } j .
$$

Note that $p_{i}(\mathbf{r}) \in[0,1]$ and $p_{i}(\mathbf{r})=1$ if firm $i$ does not default. Firm $i$ defaults if the actual value of its assets $\sum_{j \neq i} p_{j}(\mathbf{r}) a_{j i}+r_{i}$ falls below the value of its liabilities $\sum_{j \neq i} a_{i j}+l$. As shown by Eisenberg and Noé (see also AOT and GY), in the environment considered there is (generically) a unique solution of system (4) for a vector $p(\mathbf{r})=\left(. ., p_{i}(\mathbf{r}), ..\right)$. This solution is a continuous function of $\mathbf{r}$; it is however a nonlinear function of $\mathbf{r}$, more precisely it is piecewise linear. Consider for instance the case where only firm $i$ defaults: we have $p_{i}(\mathbf{r})=\left(\sum_{j \neq i} a_{j i}+r_{i}\right) /\left(\sum_{j \neq i} a_{i j}+l\right)$ and $p_{j}(\mathbf{r})=1$ for all $j \neq i$.

In the situation considered by AOT and GY the value of the firms' assets net of their internal liabilities is then given by

$$
v=f(A ; \mathbf{r})=\mathbf{r}+\left(A^{T}-A\right) p(\mathbf{r})
$$

\subsection{Contagion}

The environment presented in the previous section can be used to study how the presence of linkages among firms may generate contagion, that is, shocks hitting one firm may propagate through the whole system and possibly generate widespread default. More specifically, what we mean by a shock hitting firm $i$ is that $r_{i}<R$; the size of the shock is then given by the magnitude of $s_{i}$. The mechanism of contagion may then vary with the nature of the linkages among firms. In CGV it occurs because the presence of linkages implies that firms are exposed to the same shocks ${ }^{6}$, through a correlation in their portfolios. The same is true in EGJ, but in this case there is also an element of a domino effect, as the default of one firm constitutes an additional negative shock for the firms with whom the firm is linked.

\footnotetext{
${ }^{5}$ In AOT external liabilities are assumed to be senior to internal liabilities. This has only minor qualitative implications, that we discuss later on.

${ }^{6}$ E. Lazear, in http://online.wsj.com/news/articles/SB10001424052970203554104577003924075089102, refers to this as the 'popcorn mechanism'.
} 
A similar domino effect constitutes the only contagion mechanism in AOT and GY. ${ }^{7}$ The analysis in what follows will show however that the effect of the pattern of linkages on contagion, and more generally on the way in which shocks propagate through the system, does not depend in a fundamental way on the mechanism which generates contagion.

Following the papers mentioned above, we address this issue by considering first the case where all the $N$ firms are connected, either directly or indirectly. The presence of such connections implies that all firms in the system will be affected, to various degrees, by a shock hitting any one firm, and for sufficiently large shocks, one or more firms will default. We also restrict our attention to regular networks, where each firm is equally exposed to other firms in the system, that is, $\sum_{j \neq i} a_{j i}=\sum_{j \neq i} a_{i j}$ for all $i$, and the pattern of exposure is also the same (the matrix $A$ is symmetric). In such a situation, the identity of the firm directly hit by the shock does not matter, and we can take it, w.l.o.g., to be firm 1 .

More precisely, following EGJ we can compute the minimal size of the shock $s(j)$ hitting firm 1 such that $j \in\{1, . ., N\}$ firms default ${ }^{8}$. Let $\mathbf{r}_{j}$ be the vector with elements $(R-$ $s(j), R, . ., R)^{T}$ such that $f\left(A ; \mathbf{r}_{j}\right)$ has $j$ components less or equal to $l$ (at least one of them equal) and the other $N-j$ strictly greater than $l$. This vector is uniquely defined, except in the discontinuous framework of EGJ. Hence a shock of size $s(j)+\varepsilon$, for $\varepsilon$ small, will lead to the default of $j$ firms. Studying how the values $s(1), \ldots, s(N)$ vary with $A$, that is, with the pattern of linkages among the firms, allows to see how well different network structures do in limiting the extent of contagion. Again following the papers mentioned above, we focus most of our attention on the 'extreme' cases - as far as the density of connections is concerned - of a complete network structure, where each firm is directly linked to any other firm in the system, and of the (one-directional) ring network, where each firm is directly linked to only one other firm in the system.

We show in what follows that, with a complete network structure, either only one firm defaults (the one directly hit by the shock), or all firms default. In contrast, with the ring network structure we may have different thresholds for the size of the shocks leading to $1,2, . ., N$ firms defaulting. We will then compare the ring and the complete structure in terms of their ability to limit defaults in the system and see which role the form of $f($.$) ,$ that is, whether it is linear, piecewise linear or discontinuous, as discussed in the previous section, plays in this comparison.

Let us now be more precise. In the CGV and EGJ framework, the complete and the ring network are described, ${ }^{9}$ respectively, by the following specifications of the matrix $A$ :

\footnotetext{
${ }^{7}$ Another mechanism of contagion sometimes mentioned in the literature (see, e.g. Cifuentes et al. (2005)) is the one occurring via the fire sales of the assets of defaulting firms, which depress the value of other firms.

${ }^{8}$ We assume that $R>l$ so that in normal circumstances no firm defaults.

${ }^{9}$ See the Appendix for details.
} 


$$
A^{C}=\left[\begin{array}{cccc}
\alpha & \frac{1-\alpha}{N-1} & \cdots & \frac{1-\alpha}{N-1} \\
\frac{1-\alpha}{N-1} & \alpha & \cdots & \frac{1-\alpha}{N-1} \\
\vdots & \vdots & \ddots & \vdots \\
\frac{1-\alpha}{N-1} & \frac{1-\alpha}{N-1} & \cdots & \alpha
\end{array}\right], A^{R}=\left[\begin{array}{cccc}
\alpha & \frac{(1-\alpha)^{2}}{\left(1-\alpha^{N-1}\right)} & \cdots & \frac{(1-\alpha)^{2}}{\left(1-\alpha^{N-1}\right)} \alpha^{N-2} \\
\frac{(1-\alpha)^{2}}{\left(1-\alpha^{N-1}\right)} \alpha^{N-2} & \alpha & \cdots & \frac{(1-\alpha)^{2}}{\left(1-\alpha^{N-1}\right)} \alpha^{N-3} \\
\vdots & \vdots & \ddots & \vdots \\
\frac{(1-\alpha)^{2}}{\left(1-\alpha^{N-1}\right)} & \frac{(1-\alpha)^{2}}{\left(1-\alpha^{N-1}\right)} & \cdots & \alpha
\end{array}\right]
$$

It is natural to assume that $\alpha>(1-\alpha)^{2} /\left(1-\alpha^{N-1}\right)$, which ensures that in both cases ${ }^{10}$ each firm is more exposed to the returns of its own project than to the projects of the other firms.

Let $s^{C}(1)$ and $s^{R}(1)$ denote the minimal size of the shock leading to one firm defaulting (under the above assumption, the one directly hit by the shock), respectively for the matrix $A^{C}$ and $A^{R}$. It is immediate to see that the value of both $s^{C}(1)$ and $s^{R}(1)$ is obtained as a solution to

$$
\alpha(R-s(1))+(1-\alpha) R=l,
$$

hence $s^{C}(1)=s^{R}(1)>R-l$. This property shows that in this framework the presence of linkages to other firms allows a firm to withstand larger shocks to the returns on its project without defaulting (when $\alpha=1, s(1)=R-l$ ), that is, offers some insurance against these shocks.

With a complete structure, since all the off-diagonal terms of $A^{C}$ are the same we have $s^{C}(2)=. .=s^{C}(N)$, there is then only one other threshold, $s^{C}(N)$, defining the minimal shock leading to all the $\mathrm{N}$ firms defaulting. This is obtained from ${ }^{11}$

$$
\frac{1-\alpha}{N-1}\left(R-s^{C}(N)-\beta\right)+\left(1-\frac{1-\alpha}{N-1}\right) R=l .
$$

In contrast for the ring the off diagonal terms of $A^{R}$ have different values (and decrease with distance from the diagonal) and so we have a different threshold $s^{R}(j)$ for each $j=2, . . N$, obtained as a solution of

$$
\left(\left(\sum_{i=0}^{j-3} \alpha^{i}\right) \frac{(1-\alpha)^{2}}{\left(1-\alpha^{N-1}\right)}(R-\beta)+\frac{(1-\alpha)^{2}}{\left(1-\alpha^{N-1}\right)} \alpha^{j-2}\left(R-s^{R}(j)-\beta\right)+\left(\sum_{i=j-1}^{N-2} \alpha^{i}\right) \frac{(1-\alpha)^{2}}{\left(1-\alpha^{N-1}\right)} R+\alpha R\right)=l
$$

From the above expressions we readily obtain some properties of the pattern of contagion in the different network structures. The following result follows from the property that the off diagonal coefficients of both matrices $A^{C}$ and $A^{R}$ are decreasing in $N$.

\footnotetext{
${ }^{10}$ We have in fact $\left.(1-\alpha)\right)^{2} /\left(1-\alpha^{N-1}\right)>(1-\alpha) /(N-1)$, since this is equivalent to $1 /(N-1)<$ $1 /\left(1+\ldots+\alpha^{N-2}\right)$ and hence to $\alpha+\ldots+\alpha^{N-2}<N-2$.

${ }^{11}$ Because of limited liability there is a natural upper bound at $R$ for the admissible values of the size of the shock $s(j)$. If a solution satisfying the constraint $s(j) \leq R$ does not exist, it is not possible that $j$ firms default.
} 
RESULT 1 The minimal size of the shock leading to all firms defaulting both in the complete $s^{C}(N)$ and the ring structure $s^{R}(N)$ increases ${ }^{12}$ as $N$ increases. The same property clearly holds in the ring for all other values $s^{R}(j)$, for $N>j>1$.

Hence the larger is the system $(N)$, the more diffuse is the exposure of each firm to the returns on the projects of other firms, and the more difficult it is that a shock to the return on the project of one firm leads to contagion, with other firms defaulting. That is, larger systems are better at buffering large shocks, but when shocks are so large that they cannot be buffered, the extent of default is clearly also larger.

The key difference between the extent of contagion in the complete and ring structure is that generalized default, by all firms in the system, is harder with the ring than with the complete structure, but with the ring intermediate levels of default are also possible. Formally, recalling that $\beta$ is the cost of default for a firm, we have:

RESUlT 2 When $\beta$ equals zero or is sufficiently small, we have $s^{R}(2)<s^{C}(N)<s^{R}(N)$.

Since, as we saw $s^{C}(N)=s^{C}(j)$ for all $j>1$, this result shows that a ring structure is better able to withstand large shocks, but does worse (i.e., has a larger number of firms defaulting) for shocks of intermediate size. ${ }^{13}$ Thus when we consider the extent of contagion in ring and complete structures we face a tradeoff.

On the other hand, when the cost $\beta$ is large (that is, when the 'jumps' in the value of $f($.$) are sufficiently significant), the situation is rather different:$

RESULT 3 If $\beta$ is sufficiently large, as soon as one firm defaults all firms default as well, both for the ring and the complete structures: $s^{k}(N) \leq s^{k}(1)$ for $k=R, C$.

That is, in this case a default of any firm means an immediate default of all the firms in the system. To understand this result, and the difference with respect to Result 2, note that, when $\beta=0$, the size of the shock that needs to be absorbed by the system is given by $s$, that is, is equal to the size of the shock hitting firm 1 . In contrast, when $\beta>0$ the size of the shock the system must bear is bigger, since it also includes the cost of firms who default, and is larger the bigger is the number of defaults in the system. Thus we see clearly the amplification effect generated by the presence of default costs that must be borne (immediately) when the claims to projects' returns mutually owned by firms are given by equity. Furthermore, we can show that the property established in Result 2 is no longer valid when $\beta$ is large: generalized default is now easier in the ring than in the complete structure. This is due to the fact that contagion in the ring is triggered by the default of the direct neighbor of a firm, and this entails the obligation to face not only a share of

\footnotetext{
${ }^{12}$ This is true as long as $N$ is not so large that the system is able to withstand a shock of maximal size $(s=R)$ without having generalized default, in which case $r(N)$ is not defined.

${ }^{13}$ The result follows immediately from the fact that, as noticed in footnote 10, the largest off-diagonal term in $A^{R}$ is larger than the (common) off diagonal term of $A^{C}$, while the smallest off-diagonal term in $A^{R}$ is smaller.
} 
the shock, which declines with distance from the firm directly hit, but also a share of the default costs and these do not decrease, but actually increase with distance (as we see from (9)). ${ }^{14}$

In the AOT and GY framework regular structures can be similarly described: for the complete structure we have $a_{i j}=a_{j i}=a /(N-1)$ for some $a>0$ all $i, j$, while for the ring $a_{i i+1}=a$ for all $i$ and $a_{i j}=0$ otherwise. Focusing the attention, as in these papers, on the case where $\beta=0$, we can show that similar results to the ones obtained above still hold, in particular Results 1 and 2 are both still valid ${ }^{15}$.

RESULT 4 In the AOT/GY framework, both in the complete and the ring structure the minimal size of the shock $s^{C}(N)$ and $s^{R}(N)$ that induces default of all firms is larger the larger the number of firms $N$, and the larger the "capital buffer" $R-l$. We also have (when all claims have the same seniority), $s^{R}(N)>s^{C}(N)>s^{R}(2)$.

Hence the properties of contagion in these network structures are analogous to the case where linkages are given by cross holdings of claims. There are two main differences and we shall focus here on them. The first one is an immediate consequence of (4):

RESULT 5 In the AOT/GY framework we have $s^{C}(1)=s^{R}(1)=R-l$, while in CGV/EGJ $s^{C}(1)=s^{R}(1)>R-l$.

As already noticed, this property in CGV/EGJ readily follows from (7): it tells us that, in that framework, a firm can withstand a larger shock than the one that would trigger its default in the absence of any linkage to other firms $(R-l)$, hence the presence of linkages provides some insurance against the shock that may hit a firm. This is not true in AOT/GY as the entire value of the return $r_{i}$ generated by project $i$ shows in the asset side of firm $i$, not only a fraction $\alpha$ as in (7); we can also view this as due to the use of debt, instead of equity, in forming linkages. Hence in AOT/GY the presence of linkages among firms does not provide any insurance to a firm against the shocks hitting the returns on the firm's own project. These linkages cannot be motivated by risk sharing considerations, but from other, e.g. technological or trade related, requirements. ${ }^{16}$

The second difference is a consequence of the assumption in AOT that external claims are senior to internal claims (that is, to other firms in the network). Under that assumption we have ${ }^{17}$ :

\footnotetext{
${ }^{14}$ The details are in the Appendix.

${ }^{15}$ See the Appendix. In light of the previous discussion, it is useful to point out that the size of the shock that needs to be absorbed by the system is still given by $s$ (actually, now strictly smaller than $s$ in the presence of external claims with equal priority).

${ }^{16}$ These requirements must also preclude netting, which would otherwise be beneficial in limiting the spread of default.

${ }^{17}$ See Proposition 3 a) and b) in AOT. We also show this for completeness in the Appendix.
} 
RESULT 6 In the AOT/GY framework, when external claims are senior the minimal size of the shock inducing all firms to default is the same in the complete and the ring structure: $s^{C}(N)=s^{R}(N)$.

Since for the ring we also have, as shown in Result 4, $s^{R}(1)<s^{R}(j)<s^{R}(N)$ for $1<j<N$, for shocks of intermediate size, between $s^{R}(1)=R-l$ and $s^{R}(N)$, there are multiple failures in the ring and only a single failure in the complete structure. Thus in the environment considered by AOT there is no trade-off, and the extent of default and contagion is always (weakly) larger in the ring than the complete structure. In this respect, the ring structure is always dominated by the complete one. This is one place where the seniority of external claims in AOT makes a difference. Note the similarity of this result to Result 3, obtained in the EGJ framework when $\beta$ is large.

The extension of the above results to other network structures, exhibiting 'intermediate' densities of connections between the case of the ring and the complete structure, is discussed by AOT and EGJ. In particular, AOT consider specifications of the matrix $A$ given by convex combinations of the matrix for the ring and the complete structure, obtaining results somewhere in between those found for the ring and the complete (see, e.g., their Proposition 3 c).

\subsection{Optimality}

Once we have established the properties of networks with different densities of connections, in terms of their ability to withstand shocks of different sizes, the obvious next step is to determine the optimal network structure in a given environment. This has clearly important implications in guiding policy interventions aiming to affect the pattern of financial linkages among firms.

Regarding the notion of optimality to be considered, the papers reviewed have settled mostly for considering the number of defaulting firms as the key criterion to assess optimality. This can be justified in light of the previous observation that the default of financial firms entails a deadweight cost, given by the destruction in value of the firms' assets. Under the assumption that the cost of default is the same for each firm, the number of defaults is thus proportional to the total welfare loss in the economy.

The results of the previous section yield a number of immediate corollaries when the distribution of shocks has a single element in its support, denoted $s^{*}$. From Results 2 and 4 we have that, both in the CGV/EGJ and the AOT/GY frameworks:

RESUlt 7 a) If $s^{*} \leq s^{R}(2)$ or $s^{*}>s^{R}(N)$, then the ring and the complete networks are equivalent.

b) If $s^{R}(2)<s^{*} \leq s^{C}(N)$, then the complete network dominates the ring.

c) If $s^{C}(N)<s^{*} \leq s^{R}(N)$, then the ring network dominates the complete. 
The intuition for the result is quite simple. If the shock is small enough that all firms (possibly except the one directly hit by the shock) survive, or big enough that they all fail, independently of the network structure, there is no reason to prefer one structure over the other one. If the shock is of intermediate size there are two possibilities: it can be sufficiently large that some firms fail in the ring network, but not large enough to make more than one firm fail in the complete one, which proves then superior. Alternatively, the shock can be larger so all firms in the complete network fail, while some firms survive in the ring, so the latter is better in this case.

In the previous result we compared network structures that only differed in terms of the density of connections among firms. There are however other important aspects of the network that play an important role in determining the number of firms defaulting for different shock sizes. The first one is the amount of integration of any firm with (equivalently, its degree of exposure to) the rest of the firms in the economy, as captured by $1-\alpha$ in the EGJ/CGV framework, and by $a$ in AOT/GY. In this respect it is immediate to verify, given (7)-(9) and (10), (12), (13), that ${ }^{18}$

RESULT 8 The minimal size of the shock $s(1)$ inducing one failure increases with $1-\alpha$ in EGJ/CGV while it is invariant w.r.t. a in AOT/GY. In contrast, both in EGJ/CGV and AOT/GY $s(j)$ decreases with $1-\alpha$ (resp. a), for all $j>1$.

In other words, in the EGJ/CGV framework the presence of linkages allows firms to lower the probability of default when a shock hits them directly, providing so insurance against this event, but these linkages also enhance the probability of contagion, that is the probability that one firm default when other firms are hit. This trade-off is similar to the one observed when we discussed Result 2. The result is related to Proposition 2 in EGJ, which states that higher integration makes contagion more likely. On the other hand, as already noted after Result 5, in AOT/GY the degree of integration $a$ unambiguously increases the level of defaults in the system. Hence $a$ cannot be viewed as a meaningful policy parameter which could be varied, while $\alpha$ can.

In the analysis so far we ignored another important element that may allow to reduce the extent of contagion, and is the possibility of segmenting the system of $N$ firms into disjoint components. The possibility of segmentation may prove a more effective instrument than the weakening of connections in limiting contagion. It is in fact easy to verify that in situations like the one of Result 7.c) the segmentation of the system of $N$ firms into disjoint, complete components is typically superior to a single, ring structure. This poses the more general question of whether the choice of weaker connections but in integrated systems, versus that of denser connections in segmented systems, proves more effective in limiting the possibility of default by many firms in the presence of intermediate but large shocks. As the previous discussion makes clear, to properly analyze the pros and cons of

\footnotetext{
${ }^{18}$ The claim holds both for the complete and the ring structure, hence we omit the superscript $R$ or $C$.
} 
segmentation versus density of connection it is important to go beyond the simple shock distribution considered in Result 7 and to consider richer probability structures.

To fix attention, we follow CGV and focus on the case where shocks have a Pareto distribution. More precisely, $r=R-\rho$, where $\rho$ has support ${ }^{19}[1, \infty)$ and density $\gamma / \rho^{\gamma+1}$.Under these conditions, Propositions 1 and 2 in CGV show that:

RESUlT 9 When $\gamma<1$ the optimal network structure in CGV exhibits maximal segmentation (i.e., components of minimal size, equal to 2, in which case ring and complete networks are equivalent). When $\gamma>1$ the optimal network structure has minimal segmentation, with a single, completely connected component.

This is again easy to understand in the context of Result 7. A Pareto distribution with $\gamma<1$ exhibits fat tails, hence a sufficiently large mass of the distribution is concentrated on large shocks $s^{*}$ such that $s^{*}>s^{R}(N)$ and the only way to limit contagion and defaults in these cases is to divide the system into components of minimal size. Similarly, when $\gamma>1$, the mass of the distribution is concentrated on smaller shocks and hence it is likely to be the case that $s^{*}<s^{C}(N)$, so that a single, fully connected component allows to minimize the extent of default in the system (as in this case the opportunities for risk sharing are high). For these shock distributions, the break-up of the system into small components turns again to be more effective than a lower density of the connection among firms in limiting contagion. Similar results hold in the AOT/GY framework, as Proposition 5 in AOT shows, for example, that when shocks are large, more segmented networks always dominate the less segmented ones.

More nuanced results can be obtained with other distributions. For example, Proposition 4 in CGV states

RESUlT 10 When the shock distribution is given by a mixture of a Pareto distribution with parameter $\gamma>1$ and another Pareto distribution with parameter $\gamma^{\prime}<1$, the optimal pattern of segmentation for the completely connected structure in CGV is intermediate and symmetric, with identical components of intermediate size. ${ }^{20}$

Also, in this case, interestingly, the complete structure still dominates the ring, but Proposition 5 in CGV finds yet other, less 'regular' distributions for which a single, ring component is optimal, that is for which less dense connections are more effective than segmentation.

EGJ in turn use random networks, which in principle encompass networks with varying degree, and allow both for segmentation and different density of connections. Their main result where this is studied is their Proposition 1, where it is shown that a necessary condition for widespread contagion is that the average degree is bigger than 1, a well-known

\footnotetext{
${ }^{19}$ This distribution could be suitably truncated so as to satisfy the non-negativity constraint on $r$.

${ }^{20}$ More precisely, the result holds for an open set of values of the weights of the mixture.
} 
condition for a random network to have a giant component, ${ }^{21}$ but not too high. ${ }^{22}$ If in fact the average degree is sufficiently high one basically reverts to a complete network, which can be very robust and impedes large cascades if shocks are not too large.

Decentralized network formation may lead to departures from optimality, because of the usual disconnect between efficiency and stability of a network due to non-internalized externalities (see e.g. Goyal 2007, Jackson 2009). In the present context CGV show (Proposition 6) that these externalities may be induced by the feasibility constraint that operates on admissible configurations: in the absence of side payments, firms may then deviate to form components of individually optimal size, without internalizing that firms which are left behind will be forced to inefficiently small components.

\subsection{Heterogeneity, asymmetries and other extensions}

Even though in the previous discussion we focused our attention on the case where firms are, essentially, homogenous and on symmetric network structures, it is clearly important to extend the analysis to allow for heterogeneity among firms and asymmetries in the network. Battiston et al. (2012) show in fact that real financial networks are quite asymmetric, with a core-periphery structure, for example.

As already mentioned in the previous section, EGJ explore contagion in random networks, where asymmetries may also arise. EGJ also deal with core-periphery, homophily grouped industries, and correlated shocks in their section IV. AOT also characterize the pattern of contagion in general networks (in Proposition 7) and briefly consider the impact on contagion of the presence of firms of different sizes, studying (in Proposition 10) the threshold for contagion in that case.

CGV study the properties of the optimal network structure when firms differ for the probability distribution of the shocks they face, or for their sizes (with probability of shocks that are proportional to size). In both cases they find (Proposition 7 and 8) that default rates are minimized if firms form homogeneous components, that is, there should be positive assortative matching.

Loepfe, Cabrales and Sánchez (2013) extend CGV through numerical methods to environments with more realistic features. They find that the implications of Results 2 and 9 extend to situations with heterogeneity in the firms' size and in the distribution of linkages among firms, ${ }^{23}$ in the sense that less (more) dense networks are more robust when shocks come from unbounded distributions that put more weight on large (small) shocks even with asymmetric configurations. They also find that in the real-life network of corporate control studied by Battiston et al. (2012) the consequences of link removal (moving so to a

\footnotetext{
${ }^{21}$ See e.g. Jackson (2009) Ch. 4.

${ }^{22}$ To properly relate this result to the previous ones in this section, it is useful to point out that in a random network segmentation and density of the connections cannot be separately controlled via the degree.

${ }^{23}$ The results prove also robust to the possibility that shocks hit simultaneously more than one firm, and to other kinds of shock distributions.
} 
less dense network) on the vulnerability of the system depend on the nature of the shock distribution as suggested by the results in CGV.

Why real networks do exhibit asymmetries, and what are the consequences for contagion and optimality is an important question to address in the future. For example, risk-sharing may not be the only motive why firms are connected. They also share information, which can lead to a very centralized structure (see e.g. Guimerà et al. 2002), they provide services to one another, and joint stakes are useful in the presence of incomplete contracts (Grossman and Hart 1986, Hart and Moore 1990). This suggests that an important avenue for future research would be to study optimal network formation in environments where risk sharing and contagion are not the only tradeoffs facing the firms.

Also, a network is a complicated object and understanding the effects of shocks could thus be cognitively as well as informationally demanding. Another avenue for further research is then to properly recognize these features in studying the formation of linkages and the behavior of firms in response to shocks. This might possibly lead to effects that are similar to the market freezes discussed in section 3.2, but it needs to be explored thoroughly.

Finally, in the papers considered the arrival of a shock is independent of the network structure. But this need not be true in general, the network could influence the likelihood of a shock. To illustrate this problem, EGJ have an example (in the Online Appendix section 3 ) in which the firms manipulate the failure threshold to take advantage of neighbors. This issue needs to be explored in more depth.

\subsection{Additional literature}

Allen and Gale (2000) is a seminal contribution to the study of contagion in interconnected financial systems. They analyze a model in the Diamond and Dybvig (1983) tradition. In their model a single completely connected component is the network structure that minimizes the extent of default, but they study the tradeoffs that occur when that structure is not possible. With respect to the papers discussed in sections 2.2 and 2.3, the model considered by Allen and Gale (2000) has more detail in terms of the microfoundation of the linkages among banks and of the analysis of their decisions, but this is done at the price of a rather simplified network structure, as only four banks are present. Freixas, Parigi and Rochet (2000) consider an environment where a lower density of connections, even though it limits risk sharing, has the positive consequence of reducing the incentives for deposit withdrawal. They study an aspect of the problem we have not discussed so far, since they show that a ring induces more bank discipline than a complete structure, as the higher exposure to a single debtor increases the incentives to control moral hazard.

Cifuentes, Ferrucci and Shin (2005) explore the issue of contagion due to liquidity shocks in an Eisenberg and Noé (2001) framework where banks are required to maintain a certain

liquidity ratio, and sales by distressed banks can generate a cascade of failures through their effect on prices when the market's demand for illiquid assets is less than perfectly elastic. 
They do not explore however the impact of different structures of exposures among firms on the likelihood of contagion. Greenwood, Landier and Thesmar (2015) also explore contagion through fire sales. In addition to exploring theoretically this problem, they estimate its impact using balance sheet data for European banks, and show how these estimates can be useful to formulate policies. Another dynamic equilibrium model with cascading defaults (through fire sales of assets) is Bluhm, Faia and Krahmen (2013), which also provides a measure of systemic importance of institutions based on Shapley values.

Cohen-Cole, Patacchini and Zenou (2012) study contagion without defaults in a dynamic model with strategic lending and borrowing. They show that in equilibrium small changes in uncertainty, or behavior, can propagate through the network even without defaults, and provide a measure of total systemic risk, related to Katz-Bonacich centrality ${ }^{24}$ (Chapter XX in this handbook, by Yves Zenou, discusses other uses of Katz-Bonacich centrality in "key player" policies). They also calculate the contribution of individual banks to this measure, and verify its empirical usefulness.

Another line of literature studies the issue of contagion in the context of large, usually random, networks. In many of these papers, the approach is numerical, based on large-scale simulations. Gai, Haldane, and Kapadia (2011), for example, study a model of interbank lending and show that complexity and concentration of the network of lending relationships can amplify the fragility of the system ${ }^{25}$. Blume et al. (2011) add to these models a strategic analysis of network formation. They assume that agents benefit from the number of direct contacts they have. But they also have a cost, arising from the fact that a shock to an agent in the network is transmitted to all its contacts, direct but also indirect. They find that social optimality is attained around the threshold where a large component emerges, but individuals will generally want to connect beyond this point. This is due to the fact that agents do not internalize the effect on others of adding new channels (i.e. linkages) that facilitate the spread of shocks ${ }^{26}$. Gofman (2013) calibrates a network-based model of over-the-counter bilateral trades in the Federal funds market. He compares the calibrated architecture to nine counterfactuals and shows the presence of nonmonotonicities in the risk of contagion with respect to the maximum number of trading partners of an institution.

Finally, we should briefly mention a large empirical literature whose main objective is policy related. These papers explore which summary statistics of the network of firms' relationships are better able to predict contagion of shocks. For example, Battiston et al. (2012) propose a measure of centrality (Debtrank) that is inspired by the measure of Pagerank centrality that Google uses to rank web-pages. Denbee et al. (2011) propose a measure of centrality á la Katz-Bonacich and apply it to English data. Bonaldi, Hortaçsu and Kastl (2014) propose a measure of systemicity based on the estimation of spillovers between funding costs of individual banks.. Of particular interest in this respect is Elsinger, Lehar and

\footnotetext{
${ }^{24}$ Katz (1953), Bonacich (1987).

${ }^{25}$ See also Nier et al. (2007), Kapadia et al. (2012) and Anand et al. (2012).

${ }^{26}$ This form of inefficiency is different from the one discussed in Section 2.3.
} 
Summer (2011) who, using Austrian data, show that correlation in banks' asset portfolios is the main source of systemic risk. A related emphasis on cross-ownership and investment has been pursued as well by the literature on "balance sheet effects" to understand the Asian financial turmoil in the late 90's (Krugman 1999) as well as the current crisis (Ahrend and Goujard 2011).

\section{Informational contagion}

\subsection{Contagion between markets}

Informational contagion refers to the process whereby information about one market has an impact on another market. The study of informational contagion did not originally require the use of an explicit network, but the concept of a network is a natural framework for the study of informational contagion. Two of the earliest studies, Kodres and Pritzker (2002) [KP] and Pavlova and Rigobon (2008) [PR], illustrate the network structure that is implicit in the study of informational contagion.

$[\mathrm{KP}]$ make use of the familiar rational expectations model of financial markets, in which private information is aggregated in asset prices. They consider a model consisting of two asset markets, $A$ and $B$, and assume that traders in one market can observe the asset prices in both markets. The presence of noise traders prevents prices from revealing information perfectly. In fact, prices will reflect a combination of noise and fundamentals. The fundamental values of the assets in the two markets are affected by a combination of common and market-specific shocks. Traders in market $A$, observing changes in the prices of assets in market $B$, will try to infer information that is relevant to the valuation of assets in market $A$. In this way, an increase in prices in market $B$ may cause prices to rise in market $A$ if traders infer that the price increases in market $B$ are due to a common shock that has raised fundamental values in both markets. Since prices do not reveal information perfectly, traders in market $A$ may be fooled by price increases in market $B$ that result either from noise trading or from fundamental shocks that are irrelevant to market $A$. Thus, the spillover from market $B$ to market $A$ may be unjustified by the information originally received by traders in market $B$.

Now suppose that there are three markets, $A, B$, and $C$, and that $A$ and $B$ have a common factor, $B$ and $C$ have a common factor, but that $A$ and $C$ have no factors in common. Contagion is possible between $A$ and $B$ and between $B$ and $C$ for the usual reasons explained above. What is more surprising is that portfolio rebalancing allows financial contagion between market $A$ and market $C$. Even if there is no information asymmetry in market $B$ and no market specific shock, a shock to market $A$ will spill over to market $B$ and affect prices there. These price changes will be interpreted as the result of a shock common to $B$ and $C$ and will thereby influence prices in market $C$.

In the simplest cases, the idea of a network structure is almost superfluous, but if one 
thinks of a larger number of markets, for example, capital markets in different countries, the network concept arises quite naturally. Suppose the markets are indexed by $i=1, \ldots, m$ and the factors affecting asset values are indexed by $j=1, \ldots, n$. Each market $i$ is affected by a set of factors $J(i)$. A pair of markets $i_{A}$ and $i_{B}$ will share some common factors $J_{i_{A}} \cap J_{i_{B}}$ but each will also be affected by specific factors $J_{i_{A}} \backslash J_{i_{B}}$ and $J_{i_{B}} \backslash J_{i_{A}}$ that do not directly affect the other. Two markets $i_{A}$ and $i_{B}$ are directly connected if $J_{i_{A}} \cap J_{i_{B}}$ is non-empty. Of course, the "connection" between two markets is more complicated than the existence of an edge between two nodes in a graph, but the analogy is clear.

$[\mathrm{PR}]$, like $[\mathrm{KP}]$, use the rational expectations framework, but seek to show how informational contagion can affect economies that have no common shocks. They illustrate this idea with the Brazilian financial crisis that followed the Russian default of 1998. The link between the apparently unrelated crises in Russia and Brazil was the New York market, which was linked to both. The Russian default caused New York banks to adjust their portfolios because of institutional constraints designed to control risk exposures. This reallocation of wealth in turn caused changes in prices in the Brazilian market that signaled a bad shock. Here the network is salient: New York constitutes the centre of the network and Brazil and Russia represent two peripheral nodes.

These two papers also illustrate the difference between pure information externalities and payoff externalities. In $[\mathrm{KP}]$ there is a pure informational externality: the actions of traders in market $B$ do not affect the payoffs of traders in market $A$. The only reason that traders in market $A$ pay attention to the actions of traders (or prices) in market $B$ is because of the information those actions (or prices) reveal. In [PR], on the other hand, the actions of the traders in New York have a direct affect on the traders in Brazil, through their effect on prices, in addition to the information revealed by the change in prices. Pure information externalities are somewhat unusual in economics, but they are characteristic of models of herd behavior (Banerjee, 1992; and Bikhchandani, Hirshleifer and Welsh, 1992). In those models, a sequence of agents chooses a discrete action after observing a private signal. One can think of the sequence of agents as forming a kind of network in which agent $N+1$ observes the actions of agents $n=1, \ldots, N$, but not their private information. The discrete action chosen by the preceding agents is a coarse signal of their private information and this makes informational cascades possible, in which agents ignore their private information and follow their predecessors, and herd behavior, in which agents choose the same action indefinitely. These ideas have also been applied in market settings. Avery and Zemsky (1998) have shown that the informational role of prices can prevent the occurrence of informational cascades and incorrect herds. Cipriani and Guarino (2008a, 2008b) have found conditions under which cascades can exist in financial markets (this requires transaction costs or gains from trade) and showed that contagion may occur between asset markets (information revealed by prices in one market may cause a cascade in another market).

In $[\mathrm{KP}]$ and $[\mathrm{PR}]$, traders are assumed to observe prices in all markets, but prices are not perfectly revealing because of the presence of noise. Other writers assume that agents 
in one location can only observe a subset of other locations. Examples include Gale and Kariv (2003), Acemoglu, Dahleh, Lobel and Ozdaglar (2011), and Mueller-Frank (2013). This literature is discussed in the chapter by Golub and Sandler, in this volume.

\subsection{Contagion between banks}

Informational contagion between banks has been explored in settings that do not call for an explicit network structure. One example is the paper by Ahnert and Georg (2012), in which the release of bad information about one bank provides information about other banks, because of common asset holdings and common counterparty exposures. The paper by Ahnert and Bertsch (2014) makes use of a different type of contagion, the "wake up call." Bad information about one bank causes depositors to obtain costly information about another bank, because the depositors suspect a common shock. Even if it transpires that the second bank was not subject to a common shock, the information revealed about the second bank may, coincidentally, provoke a run. Thus, even in the absence of a common shock, there is informational contagion.

These models, like those of $[\mathrm{KP}]$ and $[\mathrm{PR}]$ are interesting because they provide an account of informational contagion between banks, but they do not make use of an explicit network. It is easy to see how these informational contagion channels could be embedded in a network framework to give a richer account of financial contagion. Two examples of financial contagion in networks are described next.

Caballero and Simsek (2013) [CS] have exploited an explicit network formulation to show how informational contagion can amplify the mechanical contagion that results from counterparty exposures. Imagine a sequence of banks, indexed by $i=1, \ldots, m$, arranged in a circular network. Bank $i$ owes one unit to bank $(i-1) \bmod m$. Banks are funded by deposits and capital (equity) and the latter allows banks to suffer some losses without failing. If bank $i$ fails completely (its assets become worthless), it cannot pay what it owes bank $i-1$. Then bank $i-1$ can pay bank $i-2$ the amount $\min \{1, e\}$, where $e$ represents the bank's equity, bank $i-2$ will be able to pay bank $i-3$ the amount $\min \{\min \{1, e\}+e, 0\}$ and so on. If bank $k$ is far enough away from bank $i$ so that $(i-k+1) e \geq 1,{ }^{27}$ it will receive the full amount and bank $k$ will not fail. Thus, a complete failure of bank $i$ only causes limited contagion within the network.

Now suppose that all banks know that some bank has failed completely but the identity of the failing bank is unknown. Then every bank will have a positive probability of being hit by the contagion and failing. In fact, if the probability distribution of any bank failing is unknown and depositors have an extreme form of uncertainty aversion, they may assume the worst case, i.e., that bank $i$ is close to the failing bank and will also fail. In effect, uncertainty aversion collapses the network, making the failed bank every bank's neighbor.

\footnotetext{
${ }^{27}$ The contagion ends at bank $k$ if bank $k-1$ can repay its debt in full and banks $k-2, \ldots, i$ cannot. In that case, bank $k-1$ can pay at most $(i-k+1) e$ and $k$ will not fail if and only if $(i-k+1) e \geq 1$.
} 
As a result, the banks take precautionary actions in the situation with uncertainty about the identity of the failing bank, such as having more liquidity available, and they do it to a considerably larger extent compared to what they do in the situation where they know the structure of the network and which bank got hit by a shock.

Notice that the CS model is related to the ones in section 2.1 (most closely, to AOT and GY), with the main substantial difference being the incomplete information about the network structure. Thus, as mentioned earlier, informational contagion in CS is an amplification mechanism, to be added to the one caused by exposure to other firms in section 2.1.

Alvarez and Barlevy (2014) [AB] have undertaken a more complex and nuanced analysis of uncertainty about the location of bad banks. In their analysis, banks are randomly assigned to locations on a network. Some of the banks are bad banks, which have received a shock that makes it impossible for them to repay their debts in full. Other banks have not received such a shock but may have counterparty exposure, directly or indirectly, to one of the bad banks. As in [CS], bank $i$ 's exposure depends on the distance between bank $i$ and the nearest bad bank. The complexity of the analysis derives partly from the generality of the networks considered and the difficulty of characterizing the distribution of good and bad banks. Each of the good banks has an investment option that can produce enough revenue to keep the bank solvent but it requires investment that has to be externally funded. The problem is that, because of the uncertainty of the location of good and bad banks, the debt overhang discourages investors from investing in the good banks. [AB] show that, under certain conditions, mandatory disclosure of banks' financial condition can permit some banks to obtain external funding and return to solvency, whereas non-disclosure ensures that none will receive the new investment they need to survive.

These two examples illustrate how financial contagion depends not only on the network structure but also on the banks' knowledge and beliefs about that structure. This suggests that providing information about or ensuring the transparency of the network structure, may be a useful regulatory tool. Of course information and transparency may also have implications for competition, as it may make tacit collusion easier. The interaction between systemic regulation, whose main objective is financial stability and competition policy, is another potentially important avenue for further research.

\section{References}

[1] Daron Acemoglu, Munther A. Dahleh, Ilan Lobel and Asuman Ozdaglar (2011) "Bayesian Learning in Social Networks," Review of Economic Studies 78, 1201-1236.

[2] Daron Acemoglu, Asuman Ozdaglar, and Alireza Tahbaz-Salehi (2014), "Systemic Risk and Stability in Financial Networks", American Economic Review, in press. 
[3] Toni Ahnert and Co-Pierre Georg (2012) "Information contagion and systemic risk," LSE Working Paper.

[4] Toni Ahnert and Christoph Bertsch (2013) "A wake-up call: information contagion and strategic uncertainty," unpublished.

[5] Rudiger Ahrend, and Antoine Goujard (2011), "Drivers of Systemic Banking Crises: The Role of Bank-Balance-Sheet Contagion and Financial Account Structure," OECD Working Paper, Vol. 71, No. 902.

[6] Fernando Alvarez and Gadi Barlevy (2014) "Mandatory disclosure and financial contagion," Federal Reserve Bank of Chicago Working Paper 2014-04.

[7] Kartik Anand, Prasanna Gai, Sujit Kapadia, Simon Brennan and Matthew Willison (2012), "A network model of financial system resilience," Bank of England Working Paper No. 458.

[8] Franklin Allen, and Douglas Gale (2000), "Financial Contagion," Journal of Political Economy 42, 1-33.

[9] Abhijit V. Banerjee (1992) "A Simple Model of Herd Behavior," The Quarterly Journal of Economics 107, 797-817.

[10] S Bikhchandani, D Hirshleifer, I Welch (1992) "A theory of fads, fashion, custom, and cultural change as informational cascades," Journal of Political Economy 100, 992-1026.

[11] Stefano Battiston, Michelangelo Puliga, Rahul Kaushik, Paolo Tasca and Guido Caldarelli (2012): "DebtRank: Too Central to Fail? Financial Networks, the FED and Systemic Risk," Scientific Reports 2, 541.

[12] Bluhm, Marcel, Ester Faia and Jan Pieter Krahnen (2013), "Endogenous Banks' Networks, Cascades and Systemic Risk," mimeo.

[13] Philipp Bonacich (1987), "Power and Centrality: A Family of Measures", American Journal of Sociology, 92, 1170-1182.

[14] Larry Blume, David Easley, Jon Kleinberg, Robert Kleinberg, and Éva Tardos (2011), "Network Formation in the Presence of Contagious Risk," Proc. 12th ACM Conference on Electronic Commerce.

[15] Pietro Bonaldi, Ali Hortaçsu and Jakub Kastl (2014), "An Empirical Analysis of Systemic Risk in the EURO-zone," mimeo.

[16] Francesco Brioschi, Luigi Buzzacchi, and Massimo G. Colombo (1989), "Risk capital financing and the separation of ownership and control in business groups," Journal of Banking \& Finance 13, 747-772. 
[17] Ricardo Caballero and Alp Simsek (2013) "Fire Sales in a Model of Complexity," The Journal of Finance 68, 2549-2587.

[18] Antonio Cabrales, Piero Gottardi, and Fernando Vega-Redondo (2013), "Risk-sharing and contagion in networks," UC3M Working papers Economics 13.

[19] Rodrigo Cifuentes, Gianluigi Ferrucci and Hyun Song Shin (2005), "Liquidity risk and contagion," Journal of the European Economic Association 3(2-3):556-566.

[20] Marco Cipriani and Antonio Guarino (2008a) "Transaction costs and informational cascades in financial markets, "Journal of Economic Behavior 6 Organization 68, $581-592$.

[21] Marco Cipriani and Antonio Guarino (2008b) "Herd Behavior and Contagion in Financial Markets," The B.E. Journal of Theoretical Economics 8, 1935-1704.

[22] Ethan Cohen-Cole, Eleonora Patacchini and Yves Zenou (2014), "Systemic Risk and Network Formation in the Interbank Market," mimeo.

[23] Amil Dasgupta (2004) "Financial Contagion through Capital Connections: A Model of the Origin and Spread of Bank Panics," Journal of the European Economic Association 2, 1049-1084.

[24] Edward Denbee, Christian Julliard, Ye Li and Kathy Yuan (2011): "Network Risk and Key Players: A Structural Analysis of Interbank Liquidity," mimeo.

[25] Douglas W. Diamond, DouglasW., and Philip H. Dybvig (1983), "Bank Runs, Deposit Insurance, and Liquidity." Journal of Political Economy 91 (5): 401-19.

[26] Laurence Eisenberg, and Thomas Noé (2001), "Systemic Risk in Financial Systems." Management Science, 47 (2), 236-249.

[27] Matthew L. Elliott, Matthew O. Jackson and Benjamin Golub (2014), "Financial Networks and Contagion" American Economic Review 104 (10): 3115-3153.

[28] Helmut Elsinger, Alfred Lehar and Martin Summer (2006), "Risk Assessment for Banking Systems", Management Science 52, 1301-1314.

[29] Mark Fedenia, James E. Hodder and Alexander J. Triantis (1994), "Cross-holdings: estimation issues, biases, and distortions," Review of Financial Studies 7, 61-96.

[30] Xavier Freixas, Bruno M. Parigi, and Jean-Charles Rochet (2000), "Systemic risk, interbank relations, and liquidity provision," Journal of Money, Credit, and Banking 32, 611-638.

[31] Prasanna Gai, Andrew Haldane, and Sujit Kapadia (2011), "Complexity, concentration and contagion." Journal of Monetary Economics 58, 453-470. 
[32] Douglas Gale and Shachar Kariv (2003) "Bayesian learning in social networks," Games and Economic Behavior 45, 329-346.

[33] Paul Glasserman and H. Peyton Young (2015), "How likely is contagion in financial networks?" Journal of Banking \& Finance 50, 383-399.

[34] Michael Gofman (2013), "Efficiency and stability of a financial architecture with toointerconnected-to-fail institutions." mimeo.

[35] Ben Golub and Evan Sadlery (2015) "Learning in Social Networks," Chapter xx in this volume.

[36] Sanjeev Goyal (2007), Connections: An Introduction to the Economics of Networks, Princeton: Princeton University Press.

[37] Robin Greenwood, Augustin Landier, and David Thesmar (2015), "Vulnerable banks" Journal of Financial Economics, in press.

[38] Sanford J. Grossman, and Oliver D. Hart. 1986. "The Costs and Benefits of Ownership: A Theory of Vertical and Lateral Integration." Journal of Political Economy, 94(4): 691-719.

[39] Roger Guimerà, Albert Díaz-Guilera, Fernando Vega-Redondo, Antonio Cabrales and Àlex Arenas (2002), Optimal network topologies for local search with congestion. Physical Review Letters, 89(24):248701.

[40] Oliver Hart, and John Moore. 1990. "Property Rights and the Nature of the Firm." Journal of Political Economy, 98(6): 1119-58.

[41] Matthew O. Jackson (2009) Social and Economic Networks, Princeton University Press: Princeton.

[42] Sujit Kapadia, Mathias Drehmann, John Elliott and Gabriel Sterne (2012), "Liquidity risk, cash-flow constraints and systemic feedbacks," Bank of England Working Paper No. 456.

[43] Leo Katz (1953), "A New Status Index Derived from Sociometric Analysis", Psychometrika 18, 39-43.

[44] Laura E. Kodres and Matthew Pritsker (2002) "Rational Expectations Model of Financial Contagion," Journal of Finance 57, 769-799.

[45] Paul Krugman, (1999), "Balance Sheets, the Transfer Problem, and Financial Crises," International Tax and Public Finance 6, 459-472. 
[46] Lasse Loepfe, Antonio Cabrales, and Angel Sánchez (2013), "Towards a Proper Assignment of Systemic Risk: The Combined Roles of Network Topology and Shock Characteristics," PLoS ONE, 8: Article number: e77526 doi:10.1371/journal.pone.0077526.

[47] Manuel Mueller-Frank (2013) "A general framework for rational learning in social networks," Theoretical Economics 8, 1-40.

[48] Erlend Nier, Jing Yang, Tanju Yorulmazer, Amadeo Alentorn (2007) "Network models and financial stability," Journal of Economic Dynamics and Control Volume 31, 20332060 .

[49] Anna Pavlova and Roberto Rigobon (2008) "The Role of Portfolio Constraints in the International Propagation of Shocks," Review of Economic Studies 75, 1215-1256.

[50] Hyun Song Shin (2009) "Securitization and Financial Stability", Economic Journal 119, 309-332.

[51] Vega-Redondo, F. (2007), Complex Social Networks, Econometric Society Monograph Series, Cambridge University Press: Cambridge.

[52] Yves Zenou (2015) "Key Players," Chapter xx in this volume. 


\section{Appendix}

We present here some further details of the argument behind some of the claims in the main text.

The expressions in (6) It is immediate to verify that the expression of $A^{C}$ is obtained from (2) with the following specification of the matrix of the cross-ownership of shares among firms ${ }^{28}$ :

$$
C=\left[\begin{array}{cccc}
0 & c /(N-1) & \cdots & c /(N-1) \\
c /(N-1) & 0 & \cdots & c /(N-1) \\
\vdots & \vdots & \ddots & \vdots \\
c /(N-1) & c /(N-1) & \cdots & 0
\end{array}\right]
$$

Similarly, $A^{R}$ is obtained from ${ }^{29}$

$$
C=\left[\begin{array}{cccc}
0 & c & \cdots & 0 \\
0 & 0 & \cdots & 0 \\
\vdots & \vdots & \ddots & \vdots \\
c & 0 & \cdots & 0
\end{array}\right]
$$

Contagion in ring and complete structures when $\beta$ is large. The relationship between $s^{C}(1)$ and $s^{C}(N)$ depends on the values of $\beta$ and $N$. By subtracting (8) from (7) we get

$$
-\alpha s^{C}(1)+\frac{1-\alpha}{N-1} s^{C}(N)=-\frac{1-\alpha}{N-1} \beta
$$

From this expression it is immediate to see that there exists some $\bar{\beta}^{C}>0$ such that $s^{C}(N)<$ $s^{C}(1)$ for $\beta>\bar{\beta}^{C}$, so that once the first default occurs, all firms default. More explicitly $\bar{\beta}^{C}$ is defined by the value of $\beta$ for which $s^{C}(1)=s^{C}(N)$, and hence satisfies

$$
\frac{1-\alpha}{N-1}\left(s^{C}(N)-s^{C}(1)\right)=\left(\alpha-\frac{1-\alpha}{N-1}\right) s^{C}(1)-\frac{1-\alpha}{N-1} \bar{\beta}^{C}=0
$$

Similarly for the ring, subtracting the expression of (9) for $j$ from that for $j-1$ we get

$$
\alpha s^{R}(j)-s^{R}(j-1)=-\alpha \beta .
$$

\footnotetext{
${ }^{28}$ The same is true for the specification in (1), when the matrix $B$ has equal off-diagonal terms.

${ }^{29}$ Similarly for (1) when

$$
B=\left[\begin{array}{cccc}
\theta & 1-\theta & \cdots & 0 \\
0 & \theta & \cdots & 0 \\
\vdots & \vdots & \ddots & \vdots \\
1-\theta & 0 & \cdots & \theta
\end{array}\right]
$$
}

and $K=N-1$. 
Proceeding by induction it is then easy to see that if $s^{R}(2)<s^{R}(1)$, we have $s^{R}(j)<$ $s^{R}(j-1)$ for all $j>2$, so the first firm defaulting brings all other firms down. Building on this observation, the threshold value $\bar{\beta}^{R}$ such that $s^{R}(1)>s^{R}(N)$ for $\beta>\bar{\beta}^{R}$ is obtained as the solution of

$$
s^{R}(1)-\alpha s^{R}(1)=\alpha \bar{\beta}^{R}
$$

Recalling that $s^{R}(1)=s^{C}(1)$, we get

$$
\frac{\bar{\beta}^{C}}{\bar{\beta}^{R}}=\frac{\alpha(N \alpha-1)}{(1-\alpha)^{2}} .
$$

Hence $\bar{\beta}^{C}>\bar{\beta}^{R}$ iff

$$
\alpha>\frac{\sqrt{4 N-3}-1}{2 N-2}
$$

and this inequality always holds, under our assumption that $\alpha>(1-\alpha)^{2} /\left(1-\alpha^{N-1}\right)$, when $N \geq 6$. Hence we can say that, provided $N$ is not too small, $\bar{\beta}^{C}>\bar{\beta}^{R}$ and so generalized default is easier in the ring than in the complete structure, that is obtains for smaller values of $\beta$ in the ring structure.

Results 1 and 2 in the AOT/GY framework (Result 4) The minimal shock $s^{C}(N)$ leading to all firms defaulting in a complete network structure in the AOT/GY environment is again obtained by considering the case where only firm 1 defaults, so that

$$
p_{1}(a+l)=a+R-s^{C}(N)
$$

while all the other firms have just enough resources to ensure they can repay $\left(p_{i}=1\right.$ for all $i \neq 1)$ :

$$
R+\left(\frac{N-2}{N-1}\right) a+a \frac{a+R-s^{C}(N)}{(N-1)(a+l)}=a+l
$$

Solving ${ }^{30}$ (10) yields the value of $s^{C}(N) .{ }^{31}$ As one would expect the threshold $s^{C}(N)$ increases (i.e. the shock has to be bigger) the larger the number of firms $N$, and the larger the "capital buffer" $R-l$ (i.e., the difference between the outside liabilities and the outside assets of a firm).

In a ring structure, denoting by $\mathbf{r}_{1}=(R-s, R, . ., R)$ the vector of realized returns where firm 1 is hit by a shock which leads it to default, we have

$$
p_{i}\left(\mathbf{r}_{1}\right)=\min \left(\frac{a p_{i-1}\left(\mathbf{r}_{1}\right)+R}{a+l}, 1\right)
$$

${ }^{30}$ Note that an admissible $(s \leq R)$ solution of $(10)$ exists iff

$$
\frac{a}{a+l} \geq \frac{R-l}{l}(N-1)
$$

\footnotetext{
${ }^{31}$ The expression is stated for the case $\beta=0$. It would not be difficult to include extra costs of reorganization with default (so that $\beta>0$ ) in this framework. The effects would be similar in this case, higher threshold for default (smaller shocks suffice to generate default) and multiple payment equilibria.
} 
for $i>1$, with $p_{1}\left(\mathbf{r}_{1}\right)=(a+R-s) /(a+l)$. It is immediate to verify that $p_{i}\left(\mathbf{r}_{1}\right) \geq p_{i-1}\left(\mathbf{r}_{1}\right)$ (with the inequality being strict as long as $i-1$ defaults). When the shock is at the threshold level $s^{R}(N)$ so that all the first $N-1$ firms default and the $N$-th firm has just enough resources to be able to repay, we also have $a p_{N-1}\left(\mathbf{r}_{1}\right)+R=a+l$ and, for all $i=2, . ., N-1$ :

$$
p_{i}\left(\mathbf{r}_{1}\right)=\frac{a}{a+l} p_{i-1}\left(\mathbf{r}_{1}\right)+\frac{R}{a+l}
$$

Hence

$$
\begin{aligned}
p_{N-1}\left(\mathbf{r}_{1}\right) & =\left(\frac{a}{a+l}\right)^{N-2}\left(\frac{a+r}{a+l}\right)+\frac{R}{a+l}\left(1+\ldots+\left(\frac{a}{a+l}\right)^{N-3}\right) \\
& =\left(\frac{a}{a+l}\right)^{N-2}\left(\frac{a+r}{a+l}\right)+\frac{R}{l}\left(1-\left(\frac{a}{a+l}\right)^{N-2}\right)
\end{aligned}
$$

so that $s^{R}(N)$ is obtained as solution of the following equation

$$
R+a\left(\left(\frac{a}{a+l}\right)^{N-2}\left(\frac{a+R-s^{R}(N)}{a+l}\right)+\frac{R}{l}\left(1-\left(\frac{a}{a+l}\right)^{N-2}\right)\right)=a+l
$$

This clearly implies that $s^{R}(N)$ is also increasing in $N$, as well as in $R-l$.

From the above we also see that $s^{R}(2)$ is obtained as a solution of

$$
R+a \frac{a+R-s^{R}(2)}{a+l}=a+l .
$$

Subtracting equation (13) from (10) yields

$$
\frac{(N-1)(a+l)+R-s^{C}(N)-l}{(N-1)(a+l)}-\left(\frac{a+R-s^{R}(2)}{a+l}\right)=0,
$$

or

$$
(N-2) l-s^{C}(N)-(N-2)\left(R-s_{2}^{R}\right)+s^{R}(2)=0
$$

and hence

$$
s^{R}(2)-s^{C}(N)=(N-2)\left(R-s^{R}(2)-l\right)<0,
$$

thus verifying the property $s^{C}(N)>s^{R}(2)$.

Finally, we verify the property $s^{C}(N)<s^{R}(N)$. From equations (10) and (12) we get

$$
\left(\frac{a}{a+l}\right)^{N-2}\left(\frac{a+R-s^{R}(N)}{a+l}\right)+\frac{R}{l}\left(1-\left(\frac{a}{a+l}\right)^{N-2}\right)=\left(\frac{N-2}{N-1}\right)+\frac{1}{N-1} \frac{a+R-s^{C}(N)}{(a+l)}
$$

and hence

$$
\left(\frac{a}{a+l}\right)^{N-2}\left(\frac{s^{R}(N)-s^{C}(N)}{a+l}\right)=\frac{R}{l}\left(1-\left(\frac{a}{a+l}\right)^{N-2}\right)-\left(\frac{N-2}{N-1}\right)+\frac{a+R-s^{C}(N)}{a+l}\left[\left(\frac{a}{a+l}\right)^{N-2}-\frac{1}{N-1}\right]
$$


Thus $s^{C}(N)<s^{R}(N)$ iff

$$
\left(\frac{R}{l}-1\right)\left(1-\left(\frac{a}{a+l}\right)^{N-2}\right)+\left(1-\frac{a+R-s^{C}(N)}{a+l}\right)\left(\frac{1}{N-1}-\left(\frac{a}{a+l}\right)^{N-2}\right)>0
$$

or, substituting for $s^{C}(N)$ and simplifying

$$
\begin{gathered}
\left(\frac{R-l}{l}\right)\left(1-\left(\frac{a}{a+l}\right)^{N-2}\right)+\left(\frac{(N-1)(R-l)}{a}\right)\left(\frac{1}{N-1}-\left(\frac{a}{a+l}\right)^{N-2}\right)>0 \\
\Leftrightarrow\left(\frac{a}{l}\right)\left(1-\left(\frac{a}{a+l}\right)^{N-2}\right)+\left(1-(N-1)\left(\frac{a}{a+l}\right)^{N-2}\right)>0
\end{gathered}
$$

The above inequality can be equivalently rewritten as

$$
a+l>\left(\frac{a}{a+l}\right)^{N-2}(a+(N-1) l) \Leftrightarrow(a+l)^{N-1}>a^{N-1}+(N-1) l a^{N-2} .
$$

Note that when $l=0(14)$ holds as equality, and its derivative w.r.t. $l$ is strictly positive for all $N>1, a>0, l \geq 0$, which establishes the validity of (14) and hence of the claimed property.

\section{Ring and complete in AOT/GY when external claims have priority (Result} 6)

In this case the expression determining $r^{C}(N)$ has to be modified as follows:

$$
R+\left(\frac{N-2}{N-1}\right) a+\frac{a+r^{C}(N)-l}{a} \frac{a}{N-1}=a+l
$$

yielding

$$
r^{C}(N)=l-(N-1)(R-l)
$$

Similarly, in a ring structure (11) has to be modified as follows

$$
p_{i}(\mathbf{r})=\min \left(\frac{a p_{i-1}(\mathbf{r})+R-l}{a}, 1\right)
$$

for $i>1$, with $p_{1}(r)=(a+r-l) / a$. At $\mathbf{r}_{N}$ we have $p_{i}\left(\mathbf{r}_{N}\right)=p_{i-1}\left(\mathbf{r}_{N}\right)+(R-l) / a$ for all $i=2, . ., N-1$ so that $p_{N-1}\left(\mathbf{r}_{N}\right)=(a+r-l) / a+(N-2)(R-l) / a$ and hence $r^{R}(N)$ is now determined as a solution of the following equation

$$
R+(N-2)(R-l)+a+r^{R}(N)-l=a+l
$$

or

$$
r^{R}(N)=l-(N-1)(R-l)=r^{C}(N) .
$$

